\title{
Annealing effects on superconductivity in $\mathrm{SrFe}_{2-x} \mathrm{Ni}_{x} \mathrm{As}_{2}$
}

\author{
S. R. Saha, N. P. Butch, K. Kirshenbaum, Johnpierre Paglione
}

Center for Nanophysics and Advanced Materials, Department of Physics, University of Maryland, College Park, MD 20742, USA

\begin{abstract}
Superconductivity has been explored in single crystals of the Ni-doped FeAs-compound $\mathrm{SrFe}_{2-x} \mathrm{Ni}_{x} \mathrm{As}_{2}$ grown by self-flux solution method. The antiferromagnetic order associated with the magnetostructural transition of the parent compound $\mathrm{SrFe}_{2} \mathrm{As}_{2}$ is gradually suppressed with increasing Ni concentration $x$ and bulk-phase superconductivity with full diamagnetic screening is induced near the optimal doping of $x=0.15$ with a maximum transition temperature $T_{c} \sim 9.8 \mathrm{~K}$. An investigation of high-temperature annealing on as-grown samples indicate that the heat treatment can enhance $T_{c}$ as much as $\sim 50 \%$.
\end{abstract}

'Keywords: superconductivity, iron-pnictides, annealing, $\mathrm{SrFe}_{2-x} \mathrm{Ni}_{x} \mathrm{As}_{2}$

The discovery of high-temperature superconductivity in new iron-based pnictide compounds has attracted much recent attention [1]. Suppression of the magnetic/structural phase transition, either by chemical doping or high pressure, is playing a key role in stabilizing superconductivity in the ferropnicitides. Oxygen-free FeAs-based compounds with the $\mathrm{ThCr}_{2} \mathrm{Si}_{2}$ type (122) structure exhibit superconductivity with $T_{c}$ as high as $25 \mathrm{~K}$ by partial substitution of Fe with other transition metal elements, e.g., $\mathrm{BaFe}_{2-x} \mathrm{Co}_{x} \mathrm{As}_{2}$ [2, 3, 4], $\mathrm{SrFe}_{2-x} \mathrm{Co}_{x} \mathrm{As}_{2}$ [5], $\mathrm{BaFe}_{2-x} \mathrm{Ni}_{x} \mathrm{As}_{2}$ [6, 7], $\mathrm{SrFe}_{2-x} \mathrm{M}_{x} \mathrm{As}_{2}(\mathrm{M}=\mathrm{Rd}, \mathrm{Ir}$, and Pd) [8]. Interestingly, in $\mathrm{BaFe}_{2-x} \mathrm{Co}_{x} \mathrm{As}_{2}$ [3, 4], the maximum $T_{c}$ is found at $x \simeq 0.17$, whereas in $\mathrm{BaFe}_{2-x} \mathrm{Ni}_{x} \mathrm{As}_{2}$, the maximum ${ }^{\prime} T_{c}$ occurs at approximately $x=0.10[6,7]$, suggesting that $\mathrm{Ni}$ substitution may indeed contribute twice as many $d$-electrons to the system as Co.

We have synthesized and studied single-crystalline $\mathrm{SrFe}_{2-x} \mathrm{Ni}_{x} \mathrm{As}_{2}$ and found that $\mathrm{Ni}$ substitution induces bulk superconductivity. Contrary to expectations framed by prior studies of similar compounds [3, 4, 6, 7], we observe a relatively low maximal $T_{c}$ value of $\sim 10 \mathrm{~K}$ in this series, centered at a $\mathrm{Ni}$ concentration approximately half that of the optimal Co concentration in $\mathrm{SrFe}_{2-x} \mathrm{Co}_{x} \mathrm{As}_{2}$ [5]. We have investigated the effect of high-temperature annealing on as grown samples. Interestingly, annealing causes an enhancement of $T_{c}$ as much as $\sim 50 \%$.

Single-crystalline samples of $\mathrm{SrFe}_{2-x} \mathrm{Ni}_{x} \mathrm{As}_{2}$ were grown using the FeAs self-flux method [1]. The FeAs and NiAs binary precursors were first synthesized by solid-state reaction of (99.999\% pure) $\mathrm{Fe} / \mathrm{Ni}$ powder with $(99.99 \%$ pure) As powders. Then FeAs and NiAs were mixed with elemental ( $99.95 \%$ pure) $\mathrm{Sr}$ in the ratio $4-2 x: 2 x: 1$ in an alumina crucible and heated in a quartz tube sealed in a partial atmospheric pressure of Ar to $1200^{\circ} \mathrm{C}$. Crystals were characterized by X-ray diffraction and wavelength-dispersive X-ray spectroscopy (WDS). Resistivity $(\rho)$ was measured with the standard four-probe ac method in a commercial PPMS and magnetic susceptibility $(\chi)$ was mea- sured in a commercial SQUID magnetometer.

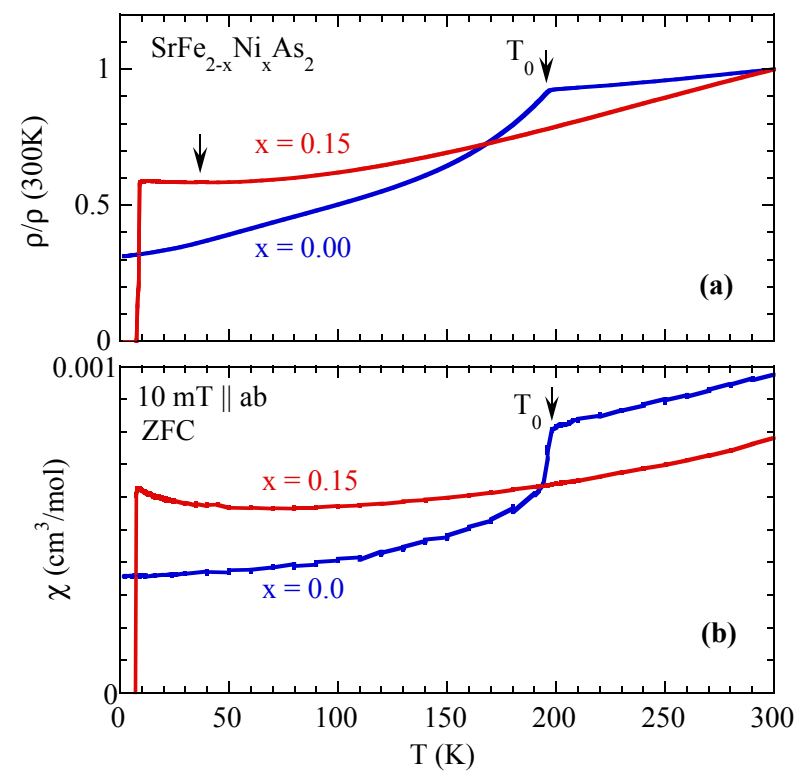

Figure 1: (a) Temperature dependence of in-plane electrical resistivity in $\mathrm{SrFe}_{2} \mathrm{As}_{2}$ and $\mathrm{SrFe}_{1.85} \mathrm{Ni}_{0.15} \mathrm{As}_{2}$ normalized to $300 \mathrm{~K}$. (b) Temperature dependence of magnetic susceptibility $\chi$ in $\mathrm{SrFe}_{2} \mathrm{As}_{2}$ and $\mathrm{SrFe}_{1.85} \mathrm{Ni}_{0.15} \mathrm{As}_{2}$ for zero-field-cooling (ZFC). The arrows indicate the position of $T_{0}$ (defined in the text).

Figure 1 (a) presents the comparison of the in-plane resistivity $\rho(T)$ between two typical single crystals of $\mathrm{SrFe}_{2} \mathrm{As}_{2}$ and $\mathrm{SrFe}_{1.85} \mathrm{Ni}_{0.15} \mathrm{As}_{2}$. As shown, $\rho(T)$ data for $\mathrm{SrFe}_{2} \mathrm{As}_{2}$ decreases with temperature from $300 \mathrm{~K}$ like a metal and then exhibits a sharp kink at $T_{0}=198 \mathrm{~K}$, where a structural phase transition (from tetragonal to orthorhombic upon cooling) is known to coincide with the onset of antiferromagnetic (AFM) order [9]. Below $T_{0}, \rho$ continues to decrease without any trace of superconductivity down to $1.8 \mathrm{~K}$. In many undoped $\mathrm{SrFe}_{2} \mathrm{As}_{2}$ samples, strain-induced superconductivity with $T_{c}=21 \mathrm{~K}$ has been 
observed [10]. However, here we present $x=0$ data for a sample with all traces of superconductivity removed by heat treatment. For $x=0.15$, which is close to optimal doping, the anomaly associated with $T_{0}$ is suppressed and transformed into a smooth minimum around $37 \mathrm{~K}$. The minimum, and hence $T_{0}$, disappears for $x>0.15$, leading to a maximum $T_{c} \sim 9.8 \mathrm{~K}$ and a dome-like superconducting phase diagram [1]. Figure 1 (b) presents the temperature dependence of the in-plane magnetic susceptibility $\chi$ in $\mathrm{SrFe}_{2} \mathrm{As}_{2}$ and $\mathrm{SrFe}_{1.85} \mathrm{Ni}_{0.15} \mathrm{As}_{2}$ crystals. The overall behavior of $\chi(T)$ for $x=0$ shows a modest temperature dependence interrupted by a sharp drop at $T_{0}$. The low-field $\chi(T)$ data at low temperatures presented here does not show any increase like that in Ref. [9], indicating both good sample quality (i.e., minimal magnetic impurity content) and no indication of strain-induced superconductivity [10]. For $x=0.15$, the large step-like feature at $T_{0}$ disappears and bulk superconductivity is induced (clearly shown in Fig. 2).

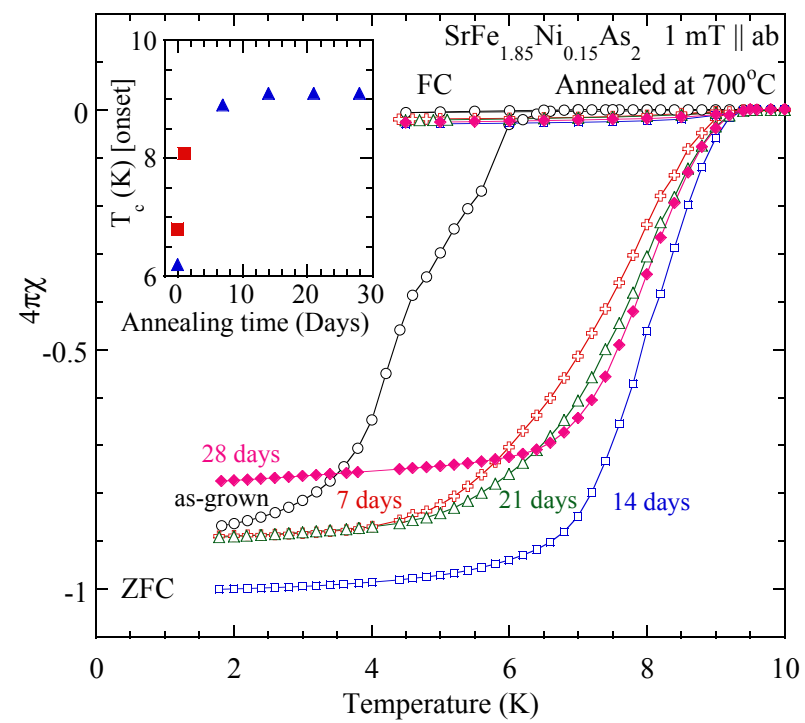

Figure 2: Volume magnetic susceptibility in $\mathrm{SrFe}_{1.85} \mathrm{Ni}_{0.15} \mathrm{As}_{2}$ sample measured before (circles) and after annealing a sample at $700^{\circ} \mathrm{C}$ for 7 days (pluses), 14 days (squares), 21 days (triangles), and 28 days (diamonds). The lines are guides through the data points. The inset shows the annealing time dependence of $T_{c}$ for this sample (filled triangles). The enhancement of $T_{c}$ in a second piece of sample annealed for 1 day is also plotted (filled squares).

We have investigated the effect of high temperature annealing on single crystals of $\mathrm{SrFe}_{2-x} \mathrm{Ni}_{x} \mathrm{As}_{2}$ and found a rather dramatic $10-50 \%$ enhancement in the value of $T_{c}$. This enhancement is reflected in the full diamagnetic screening and is therefore a bulk phenomenon. Figure 2 shows the effect of annealing on the superconducting transition detected in $\chi(T)$ of one $\mathrm{SrFe}_{1.85} \mathrm{Ni}_{0.15} \mathrm{As}_{2}$ annealed at $700^{\circ} \mathrm{C}$ after wrapping with $\mathrm{Ta}$ foil and sealing in a quartz tube under partial atmospheric pressure of Ar. Annealing for 7 and 14 days enhances the $T_{c}$ (onset) from $\sim 6.2$ in the as-grown sample to $\sim 8.9 \mathrm{~K}$ and $\sim 9.2 \mathrm{~K}$, respectively, with the sharpening of the transition. Annealing for 21 and 28 days does not enhance the $T_{c}$ further, while it gradually reduces the superconducting volume fraction, indicating 14 days as the optimal annealing time. The inset shows the annealing time dependence of $T_{c}$. Enhancement of $T_{c}$ due to annealing of as-grown $\mathrm{SrFe}_{2-}{ }_{-} \mathrm{Ni}_{x} \mathrm{As}_{2}$ (for several values of $x$ ) for 1 day at $700^{\circ} \mathrm{C}$ has been found both in $\rho(T)$ and $\chi(T)$ measurements [1]. Such an enhancement of $T_{c}$ could be an indication of improved crystallinity due to release of residual strain, and/or improved microscopic chemical homogeneity of Ni content inside the specimens, thereby optimizing the stability of superconductivity.

A similar annealing effect was reported in $\operatorname{LnFeOP}(\mathrm{Ln}=\mathrm{La}$, $\mathrm{Pr}, \mathrm{Nd}$ ) single crystals, where a heat treatment in flowing oxygen was also found to improve superconducting properties [11]. It is further noteworthy to report that some as-grown crystals of $\mathrm{SrFe}_{2-x} \mathrm{Ni}_{x} \mathrm{As}_{2}$ for $x<0.16$ (except $x=0.10$ ) show what looks to be a partial superconducting transition near $20 \mathrm{~K}$ that is completely removed by heat treatment [1]. Although it is tempting to posit that $20 \mathrm{~K}$ is a possible value for optimal $T_{c}$ in this series of Ni-substituted compounds, note that aside from the enhancement of $T_{c}$ as mentioned above, the removal of this feature is the only change observed in measured quantities imposed by annealing: neither the normal state resistivity nor magnetic susceptibility show any change after annealing. Furthermore, susceptibility does not show any indication of diamagnetic screening in the as-grown samples at $20 \mathrm{~K}$. Because the $20 \mathrm{~K}$ kink is removed with heat treatment, and, moreover, is always found to be positioned near the same temperature, we believe this feature may be connected to the strain-induced superconductivity found in undoped $\mathrm{SrFe}_{2} \mathrm{As}_{2}$ [10]. However, note that only a mild 5 minute heat treatment of $300^{\circ} \mathrm{C}$ removes the partial volume superconductivity in $\mathrm{SrFe}_{2} \mathrm{As}_{2}$, while a substantially higher temperature $\left(700^{\circ} \mathrm{C}\right)$ is required to remove this feature in $\mathrm{SrFe}_{2-x} \mathrm{Ni}_{x} \mathrm{As}_{2}$. If the two phenomena are related, it is possible that internal strain is stabilized by the chemical inhomogeneity associated with transition metal substitution in $\mathrm{SrFe}_{2-x} \mathrm{Ni}_{x} \mathrm{As}_{2}$ thus requiring higher temperatures to remove. More systematic studies of the effect of annealing on $\mathrm{SrFe}_{2-x} \mathrm{Ni}_{x} \mathrm{As}_{2}$ are under way to investigate the microscopic change in the sample.

In summary, single crystals of the Ni-substituted series $\mathrm{SrFe}_{2-x} \mathrm{Ni}_{x} \mathrm{As}_{2}$ were successfully synthesized. The magnetostructural order is suppressed and bulk superconductivity arises near the optimal doping $x=0.15$ with a $T_{c}$ value reaching as high as $\sim 9.8 \mathrm{~K}$. Interestingly, annealing treatments of as-grown single crystals result in a rather strong enhancement of the superconducting transition across this range of $x$, with $\sim 50 \%$ increase in $T_{c}$ values for $x=0.15$ for an optimal annealing time of 14 days.

The authors acknowledge P. Y. Zavalij and B. W. Eichhorn for experimental assistance, and R. L. Greene for useful discussions. N.P.B. acknowledges support from CNAM.

\section{References}

[1] S. R. Saha et al., Phys. Rev. B. 79, 224519 (2009); references therein.

[2] A. S. Sefat et al., Phys. Rev. Lett. 101, 117004 (2008).

[3] J.-H. Chu et al., Phys. Rev. B 79, 014506 (2009).

[4] N. Ni et al., Phys. Rev. B 78, 214515 (2008).

[5] A. Leithe-Jasper et al., Phys. Rev. Lett. 101, 207004 (2008).

[6] L. J. Li et al., New J. Phys. 11, 025008 (2009). 
[7] P. C. Canfield et al., Phys. Rev. B. 80, 060501(R) (2009).

[8] F. Han et al., Phys. Rev. B. 80, 024506 (2009).

[9] J.-Q. Yan et al., Phys. Rev. B 78, 024516 (2008).

[10] S. R. Saha et al., Phys. Rev. Lett. 103, 037005 (2009).

[11] R. E. Baumbach et al., New J. Phys. 11, 025018 (2009). 